\title{
The spatial-temporal evolution of coherent structures in log law region of turbulent boundary layer
}

\author{
Xiao-Bo Zheng · Nan Jiang
}

Received: 28 July 2014 / Revised: 18 October 2014 / Accepted: 14 November 2014

(C)The Chinese Society of Theoretical and Applied Mechanics and Springer-Verlag Berlin Heidelberg 2015

\begin{abstract}
The spatial-temporal evolution of coherent structures (CS) is significant for turbulence control and drag reduction. Among the CS, low and high speed streak structures show typical burst phenomena. The analysis was based on a time series of three-dimensional and three-component (3D-3C) velocity fields of the flat plate turbulent boundary layer (TBL) measured by a Tomographic and Time-resolved PIV (Tomo TRPIV) system. Using multi-resolution wavelet transform and conditional sampling method, we extracted the intrinsic topologies and found that the streak structures appear in bar-like patterns. Furthermore, we seized locations and velocity information of transient $\mathrm{CS}$, and then calculated the propagation velocity of CS based on spatial-temporal cross-correlation scanning. This laid a foundation for further studies on relevant dynamics properties.
\end{abstract}

Keywords Log law region in TBL - Spatial-temporal crosscorrelation - Tomographic TRPIV - Coherent structures · Low and high speed streaks - Multi-resolution wavelet decomposition

The project was supported by the National Natural Science Foundation of China (11332006, 11272233, and 11411130150) and the National Basic Research Programm of China (2012CB720101).

X.-B. Zheng · N. Jiang (四)

Department of Mechanics,

Tianjin University, 300072 Tianjin, China

e-mail: nanj@tju.edu.cn

N. Jiang

Tianjin Key Laboratory of Modern Engineering Mechanics, 300072 Tianjin, China

The State Key Laboratory of Nonlinear Mechanics, Institute of Mechanics, Chinese Academy of Sciences, 100190 Beijing, China

\section{Introduction}

It is generally accepted that coherent structures (CS) are multi-scale quasi-ordered fluctuating structures [1-4] in a spatial-temporal self-organizing mechanism $[5,6]$. They play a key role in turbulent boundary layer (TBL). No matter in the inner or the outer regions of TBL, there exist CS in the most intensive mode [7]. The CS in log law region have been observed in hot wire anemometry (HWA) measurement combined with flow visualization [8] and in two dimensional PIV (2D-PIV) experiments [9]. Low and high speed streak structures are basic CS inducing intensive intermittent events like sweep and ejection $[6,10,11]$ (corresponding to the second and the forth quadrants in the stream-wise and wall-normal fluctuating velocity phase plane, namely Q2 and Q4), and are closely linked to the production, maintenance, evolution and development of hairpin-like eddies and other structures [12]. All of the structures mentioned above have significant impacts on physical characteristics like friction drag, heat transfer rate and so on, and on the mechanism of mass transportation, momentum exchange and energy cascade in TBL [13].

The Tomo TRPIV is a non-contact, full-field and quantitative measurement [14] that inherits the features of 2DPIV and TRPIV. It has advantages over flow visualization, HWA and laser doppler anemometry (LDA). In addition to the high time-resolution [15], it is more suited to study the spatial-temporal evolution of CS. At present, there are mainly Tomo TRPIV experimental studies in TBL that can gain statistical properties from 3D-3C velocity fields, directly reflecting evolution of TBL flow by autocorrelation of velocity vectors and conditional sampling the turbulent signals based on Q2 or Q4 events [16-18]. Although some direct numerical simulation work has introduced new methods and models [19,20], those Tomo TRPIV experimental studies on dynamic space-time evolution of CS that takes the multi-scale nature of turbulence into account are still limited in number. 
In this paper, based on 3D-3C velocity fields, we used multi-resolution wavelet decomposition and improved conditional sampling method aiming at burst events $[6,10,18]$ to discuss the statistical properties of coherent structures dependent on the distance from wall. Then we applied the spatial phase average method [17] and extracted the bar-like intrinsic topologies of streak structures. Finally, the spatialtemporal cross-correlation scanning at fluctuating velocity fields with intrinsic topology as the template helped us seize the location and velocity information of the transient CS. This provided a method to calculate the propagation velocity of CS and laid a foundation for further studies on relevant dynamics properties like deformation. The studies on statistics properties and dynamic spatial-temporal evolution of CS, especially the migrating features, would enrich the Hierarchical Structure Theory, premise the turbulent models conforming to multi-scale and make the turbulence prediction and control by CS feasible [21,22].

\section{Experimental setup and flow field validation}

The experiments were conducted in a water tunnel with the temperature kept constant. The TBL flow was developing along a flat acrylic glass plate mounted vertically in the water tunnel. A spanwise attached zig-zag band on elliptic leading edge of the plate could accelerate the transition of boundary layer, meanwhile, nearly zero pressure gradient was achieved by adjusting flap on trailing edge of the plate. The light was scattered by polyamide seeding particles with $56 \mu \mathrm{m}$ mean diameter.

The experimental apparatus set-up is shown in Fig. 1 with the absolute object space coordinate system $O_{1} X Y Z$ and relative coordinate system $O_{2} x y z$. $O_{1} X Y Z$ is located in physical space with $O_{1}$ at leading edge of the plate, while $O_{2} x y z$ is located in 3D particle image space. $X \& x$ are in the streamwise direction, $Y \& y$ are in the spanwise direction, and $Z \& z$ are in the wall-normal direction. Both the coordinate systems follow the right-hand rule.

The basic statistical properties of the flow field were firstly tested by the low-repetition rate and high-resolution two component PIV (2C-PIV), and its spanwise center was at $X=2090 \mathrm{~mm}$ downstream the leading edge. The flow field is a fully developed TBL with zero pressure gradient, which is confirmed by the standard logarithm-law mean velocity profile in Fig. 2. The other results are listed in Table 1. In the second step, a high-repetition rate Tomo TRPIV system was applied. The measurement system had 5 CMOS cameras posed in the form of Scheimpflug with another CMOS aligned with its optical axis normal to the mean flow. It reconstructed the $3 \mathrm{D}$ particle light intensity distributions by means of the multiplicative algebraic reconstruction technique (MART) tomographic algorithm specialized for Tomo PIV [23]. The system contained a volume selfcalibration link which is necessary for improving reconstruction performance [24] and reducing the ghost particles before measurement. Then local 3D cross-correlations with an iter- ative multi-grid volume deformation scheme were adopted to analyze the particle image. A series of 1023 instantaneous $3 \mathrm{D}-3 \mathrm{C}$ velocity volumes for approximate $2 \mathrm{~s}$ with each time step of $2 \mathrm{~ms}$ increment was obtained, and there were totally $92 \times 99 \times 22(x \times y \times z)$ measurement points located every $0.687 \mathrm{~mm}(\mathrm{~d} x=\mathrm{d} y=\mathrm{d} z=0.687 \mathrm{~mm}=15 \mathrm{WU}, \mathrm{WU}$ is the nondimensional wall unit obtained with the friction velocity $u_{\tau}$ and the kinematic viscosity coefficient $v$ in all directions in space. More details about experimental equipments, image shoot and process such as the functional parameter of the laser device and CMOS cameras, the overlap rate and moving step length of the interrogation volume can be found in the paper [17].

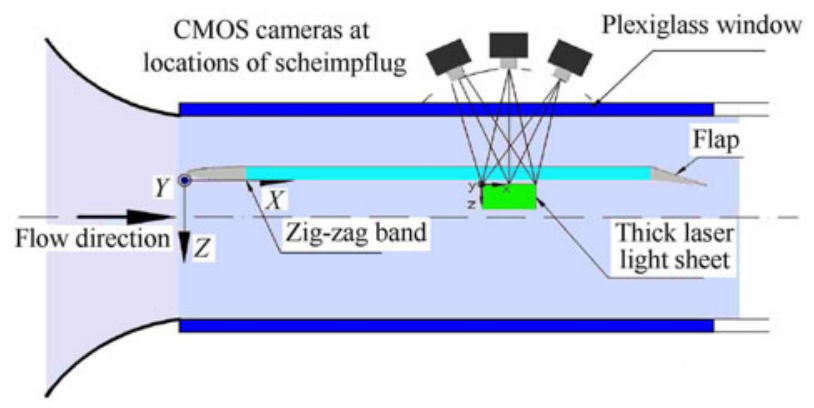

Fig. 1 Schematic of the experimental set-up

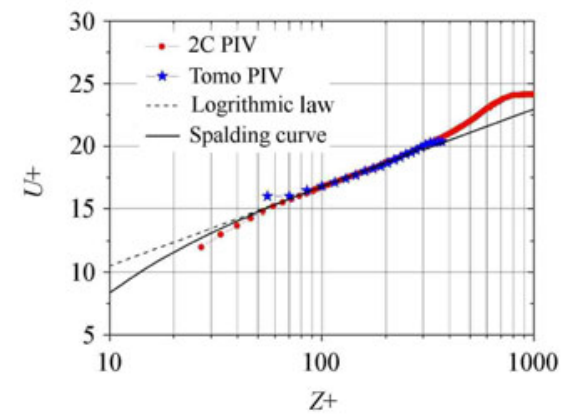

Fig. 2 Profile of the streamwise mean velocity

Table 1 Basic fluid field properties of the TBL

\begin{tabular}{ll}
\hline Quantity & Value \\
\hline Free-stream velocity $U /\left(\mathrm{m} \cdot \mathrm{s}^{-1}\right)$ & 0.53 \\
Free-stream turbulence level $T_{1}$ & $<0.5 \%$ \\
Boundary-layer thickness $\delta / \mathrm{mm}$ & 38.1 \\
momentum loss thickness $\theta / \mathrm{mm}$ & 4.64 \\
Friction velocity $u_{\tau} /\left(\mathrm{m} \cdot \mathrm{s}^{-1}\right)$ & 0.0219 \\
Friction coefficient $C_{\mathrm{f}}$ & 0.00345 \\
Reynolds number $R e_{\tau}=u_{\tau} \delta / v$ & 834 \\
Reynolds number $\operatorname{Re}_{\theta}=U \theta / v$ & 2460 \\
Taylor spatial micro-scales $\lambda /(\mathrm{WU})$ & $\sim 20-50$ \\
Kolmogorov time scales $\tau_{\eta} / \mathrm{ms}$ & $\sim 25-44$ \\
\hline
\end{tabular}


The dimension analysis shows that the Kolmogorov time scale can be expressed as $\tau_{\eta}=(v / \varepsilon)^{1 / 2}$, therein $\varepsilon$ is mean dissipation rate of turbulent kinetic energy and $v$ is kinematic viscosity coefficient. The scale of $\tau_{\eta}$ indicates that the fluctuating kinetic energy is transformed into thermal energy by the viscous movement. The Taylor spatial micro-scale $\lambda$ is the length scale of the smallest eddies identifiable and produced in turbulence. Before acquiring $\lambda=\left(-f^{\prime \prime}(0)\right)^{-1 / 2}=\left\{r^{2} /[2(1-f(r))]\right\}^{1 / 2}$, we calculate the autocorrelation coefficient of longitudinal fluctuating velocity sequence, $f(r)=\overline{u^{\prime}(x+r) u^{\prime}(x)} / \overline{u^{\prime 2}(x)}$. According to the analysis results listed in Table 1 about characteristic length scale [18], the distance of $15 \mathrm{WU}$ between the nearest two measuring points is less than the Taylor differential scale describing the size of the smallest eddies that could be detected. Therefore, the space resolution of our Tomo TRPIV experiment is refined enough to meet the identification requirements of varying scale coherent structures in log law region. Since the flow quantity and actuating pressure are kept constant, the TBL flow is a time stationary process and the ensemble average value of velocity can be replaced by the time average value according to the ergodic assumption. The plate employed by the experiments is wide enough in spanwise direction, so the velocity fields can be treated as homogeneous along streamwise and spanwise directions. Hence, we can achieve streamwise velocity profile of Tomo TRPIV by time averaging and spanwise \& streamwise spatial averaging. As shown in Fig. 2, this velocity fields are at wallnormal locations $55<Z+<369$ that belong to the $\log$ law region. Although the profile deviates slightly from the profile obtained by $2 \mathrm{C}$-PIV at $Z+=55 \& 369$ for the reason that the impacts of ghost particles are biggest at the border of the scattered light intensity Tomo reconstruction volume [8], the two profiles overlap well in the vast majority of shot flow field where we would study. Due to the hardware restriction, the dimension of shot flow field should be chosen appropriately to achieve high-quality Tomo particle images. However, the setup $\left(32^{3}\right.$ voxel final interrogation volume size with $75 \%$ overlap and $2 \mathrm{~ms}$ time delay) helps us attain a satisfactory $3 \mathrm{D}$ cross-correlation result about the dynamic evolution of velocity fluctuations in log law region, which is poor for cases in other regions of TBL. Therefore, the CS in log law region is chosen as the study object.

\section{Experimental data process and analysis}

\subsection{Spatial scale confirmation}

The CS in TBL own multi-scale nature in space-time, and so far we have grasped more fully understanding of their temporal multi-scale with high time resolved HWA [25,26]. Their spatial multi-scale nature is manifested as turbulent structures in various sizes located self-similarly in TBL. For 3D$3 \mathrm{C}$ velocity fields in finite physical space, the wavelet analysis owns locality both in physical space and in wave number space. It is an effective means to confirm the spatial scale of CS.
In a fast algorithm of the multi-resolution wavelet decomposition and reconstruction - the Mallat pyramidal algorithm $[27,28]$, the scaling coefficient vector of Scale $n$ $\boldsymbol{c}(n)$ is filtered by the band-pass filter $\left\{\boldsymbol{H}_{0}, \boldsymbol{H}_{1}\right\}$ and then goes through downsampling. Finally, the scaling coefficient vector and wavelet coefficient vector of Scale $n-1$ $\boldsymbol{c}(n-1) \& \boldsymbol{d}(n-1)$ are achieved via the flow path shown in Fig. 3. The bigger the scale index $n$, the shorter the corresponding space characteristic length. Meanwhile the characteristic lengths in two adjacent scales meet the dyadic relationship. Due to the restriction of gauging point distribution, the fluctuating velocity signals can be decomposed into 6 scales in both streamwise and spanwise directions. The physical characteristic lengths are respectively $960 \mathrm{WU} / 480 \mathrm{WU} / 240 \mathrm{WU} / 120 \mathrm{WU} / 60 \mathrm{WU} / 30 \mathrm{WU}$, when the streamwise scale index $n_{x}$ and the spanwise scale index $n_{y}$ are set to $1 / 2 / 3 / 4 / 5 / 6$.

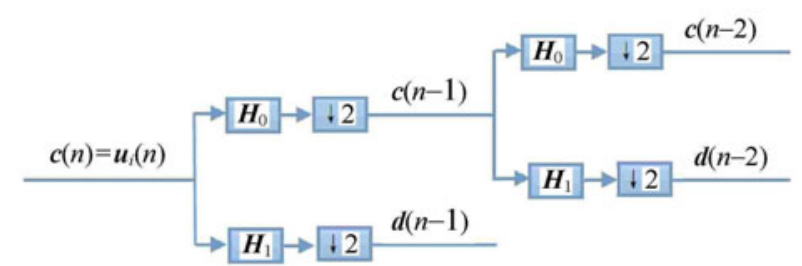

Fig. 3 Flow-process diagram of the wavelet decomposition

We choose the Haar filter which is the first-order Daubechies filter with orthogonality and non-redundancy. On the other hand, its linear phase property makes the reconstruction simple and feasible. Through the first-order Daubechies filter

$$
\begin{aligned}
& \boldsymbol{H}_{0}=\left[\begin{array}{ll}
1 / \sqrt{2} & 1 / \sqrt{2}
\end{array}\right], \\
& \boldsymbol{H}_{1}=\left[\begin{array}{ll}
1 / \sqrt{2} & -1 / \sqrt{2}
\end{array}\right],
\end{aligned}
$$

the streamwise, spanwise and wall-normal fluctuating velocities $u^{\prime} / v^{\prime} / w^{\prime}$ are respectively counted as the scaling coefficients of the first level and are decomposed along streamwise and spanwise directions in consideration of the statistics homogeneity in these two directions. In practical terms of the streamwise wavelet decomposition of a certain fluctuating velocity component $u_{i}\left(u_{i}=u^{\prime} / v^{\prime} / w^{\prime}\right)$, the wavelet coefficient vector $\boldsymbol{d}_{u_{i}}\left(n_{x}, Y, Z+\right)$ and its 2 -norm wavelet energy

$E_{u_{i}}\left(n_{x}, Y, Z+\right)=\left\|\boldsymbol{d}_{u_{i}}\left(n_{x}, Y, Z+\right)\right\|_{2}^{2}$

are functions of structure spatial scale $n_{x}$, spanwise coordinate $Y$ and wall-normal coordinate $Z+$ scaled with wall units. Then the final result $E_{u_{i}}\left(n_{x}, Z+\right)$ is achieved by spatialaveraging $E_{u_{i}}\left(n_{x}, Y, Z+\right)$ along the spanwise direction. Haar wavelet energy function turns out virtually to be the second order structure function which is coarse-grained with the concept of local averaging, and characterizes the mean turbulent energy of every turbulent structure at a certain scale [29]. The spanwise wavelet decomposition has similar procedure and is omitted here. 
The wall-normal distributions of the streamwise, spanwise and wall-normal turbulent energy $E_{u} / E_{v} / E_{w}$ are shown in Fig. 4. $E_{v}$ and $E_{w}$ are similar and increase slightly with $Z+. E_{u}$ is about 4 times of $E_{v} / E_{w}$ at $Z+=70.2$ and decreases relatively quickly with $Z+$, so that it is just a little larger than 2 times of $E_{v} / E_{w}$ at $Z+=323.5$. This result indicates that the effect of wall shear stress becomes smaller and the development of TBL flow shows the trend of isotropy with increasing $z$ in log law region.

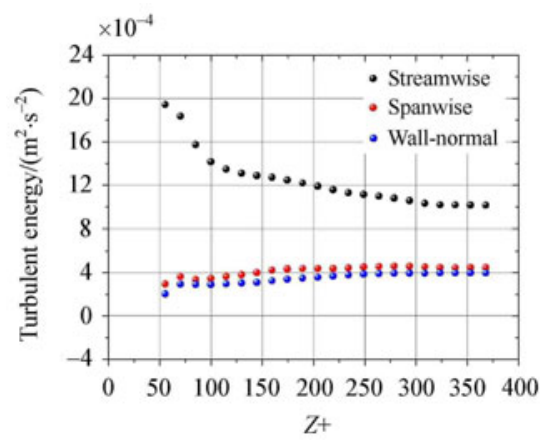

Fig. 4 Distribution of $E_{u} / E_{v} / E_{w}$ along the wall-normal direction
Figure 5 shows the percentage distributions of $E_{u_{i}}$ $\left(n_{j=x / y}, Z+\right)$ at all the 6 scales. In log law region of $Z+=$ $70.2-323.5$, there is a little difference among the scale distributions of the wavelet energy at different wall-normal locations. $E_{u^{\prime}}\left(n_{j}, Z+\right) / E_{v^{\prime}}\left(n_{j}, Z+\right) / E_{w^{\prime}}\left(n_{j}, Z+\right)$ are concentrated at Scale $n_{x}=2 / 3 / 4$ along streamwise direction and at Scale $n_{y}=3 / 3 / 4$ along spanwise direction. Considering the prominent performance of streamwise fluctuating, we confirm that the streamwise characteristic length of CS in the most intensive mode corresponds to Scale 2 (the mean wavelength of Scale 2 is $480 W U$ ), while the spanwise characteristic length corresponding to Scale $3(240 W U)$ is half of the former. Based on spatial phase averaging of streamwise Scale 2 and spanwise Scale 3, we can achieve topologies of CS that have more fluctuating energy and are more obvious in the multiscale turbulence system. As $Z+$ increases, the $\mathrm{CS}$, whose mean spanwise distance is $100 \mathrm{WU}$ in near wall region [1], are lifted up, and finally develop into larger coherent structures with mean spanwise distance of $240 \mathrm{WU}$ in $\log$ law region. Besides, the $480 \mathrm{WU}$ mean streamwise length is in well agreement with the conclusion drawn by Adrian [9] about CS outside the near wall region.
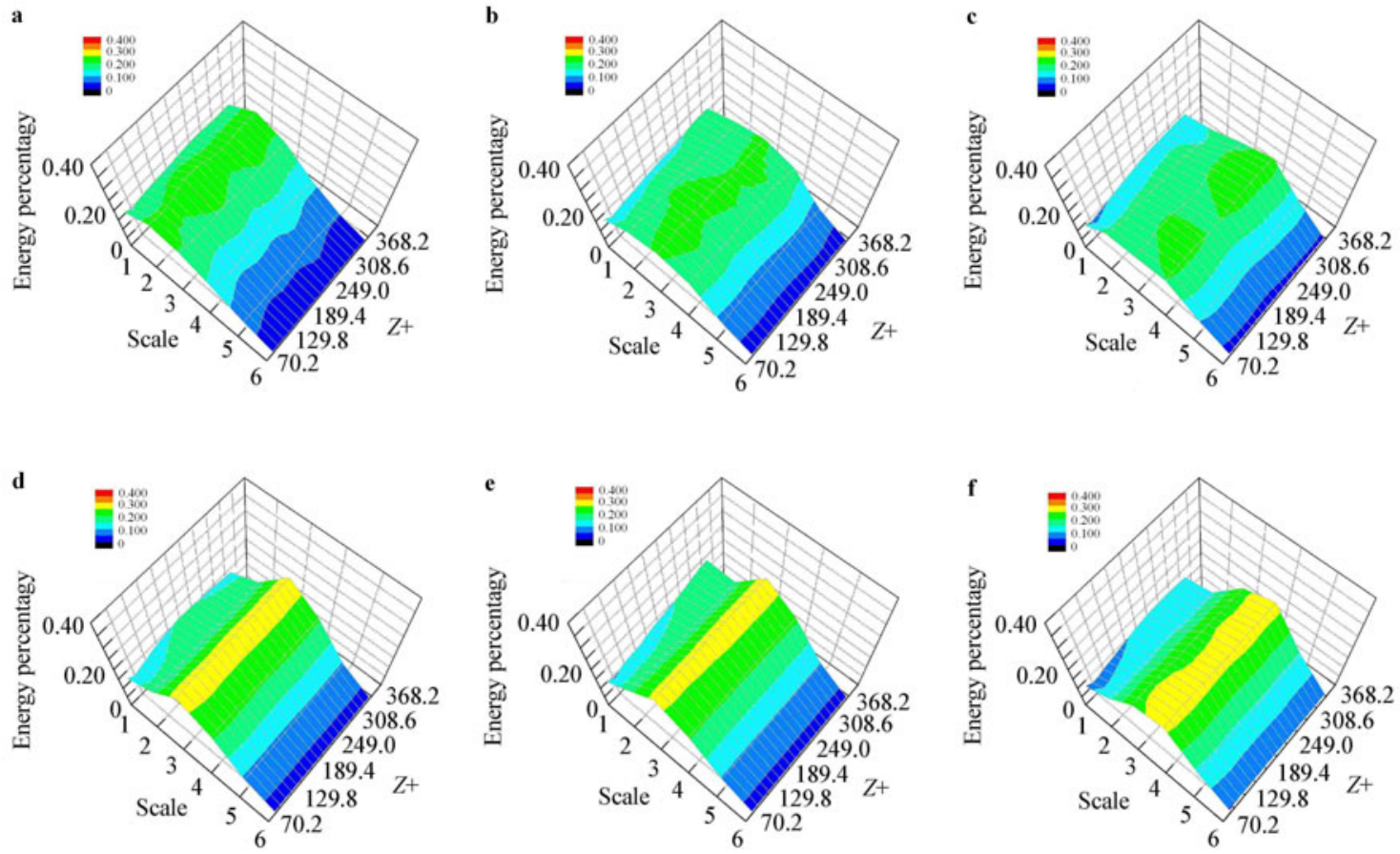

Fig. 5 a, b, c Percentage distributions of $E_{u^{\prime}}\left(n_{x}, Z+\right) / E_{v^{\prime}}\left(n_{x}, Z+\right) / E_{w^{\prime}}\left(n_{x}, Z+\right) ; \mathbf{d}$, e, f Percentage distributions of $E_{u^{\prime}}\left(n_{y}, Z+\right) /$ $E_{v^{\prime}}\left(n_{y}, Z+\right) / E_{w^{\prime}}\left(n_{y}, Z+\right)$

\subsection{Spatial location detection}

Burst process is the main production mechanism of fluctuating velocity and turbulent Reynolds stress in TBL. It is also the reason of strong intermittency. Therein, the Q2 events represent the upcast of low speed fluids that is $u^{\prime}<0$ \& $w^{\prime}>0$, while the Q4 events represent the underwashing of high speed fluids that is $u^{\prime}>0 \& w^{\prime}<0$. The instantaneous locations of CS can be identified by detecting these two kinds 
of events. The wavelet coefficient keeps correspondence with local averaged velocity structure function $[25,30]$. The spatial local averaged fluctuating velocity at Scale $n$

$u_{n}(\tau)={\overline{u^{\prime}(t)}}_{t \in\left[\tau-2^{n-1}, \tau+2^{n-1}\right]}=\frac{1}{2^{n}+1} \sum_{i=\tau-2^{n-1}}^{\tau+2^{n+1}} u^{\prime}(i)$

is used to take the place of the instantaneous fluid particle velocity in classical velocity structure function. The local averaged velocity structure function

$\delta u_{n}(\tau)=u_{n}(\tau)-u_{n}(\tau-1)$

represents the relative velocity of structures rather than fluid particles and takes the multi-scale property into consideration. $\delta u_{n}(\tau)>0$ means that the flow structure is stretching with high speed fluids in the former and low speed fluids in the latter. On the other hand, $\delta u_{n}(\tau)<0$ implies that the flow structure is compressing with low speed fluids in the former and high speed fluids in the latter.

Based on the above scale analysis (Fig. 5), we choose the local averaged velocity structure function $\delta u_{2}(\tau)$ involving 32 streamwise measurement steps to detect burst events. The indicative function for detection is set as

$I(\tau)= \begin{cases}-1(\mathrm{Q} 2), & \delta u_{2}(\tau)<0, \quad \delta u_{2}(\tau+1)>0, \quad u_{2}(\tau)<0, \\ 0, & \text { otherwise, } \\ 1(\mathrm{Q} 4), & \delta u_{2}(\tau)>0, \quad \delta u_{2}(\tau+1)<0, \quad u_{2}(\tau)>0 .\end{cases}$

Therein

$$
\begin{aligned}
& \delta u_{2}(\tau)=u_{2}(\tau)-u_{2}(\tau-1)<0, \\
& \delta u_{2}(\tau+1)=u_{2}(\tau+1)-u_{2}(\tau)>0, \\
& u_{2}(\tau)<0
\end{aligned}
$$

is the negative minimum value of mean structure velocities and can represent the center of low speed streak beside Q2 events, and

$$
\begin{aligned}
& \delta u_{2}(\tau)=u_{2}(\tau)-u_{2}(\tau-1)>0, \\
& \delta u_{2}(\tau+1)=u_{2}(\tau+1)-u_{2}(\tau)<0, \\
& u_{2}(\tau)>0
\end{aligned}
$$

is the positive maximum value of mean structure velocities and can represent the center of high speed streak beside Q4 events.

The instantaneous streamwise local averaged results $u_{2}(\tau)$ are shown in Fig. 6 . The blue isosurface $u_{2}(\tau)=$ $-0.05 \mathrm{~m} / \mathrm{s}$ represents low speed fluids and the orange isosurface $u_{2}(\tau)=0.05 \mathrm{~m} / \mathrm{s}$ represents high speed fluids. High and low speed fluids are arranged quasi-periodically in spanwise direction. Their instantaneous spanwise distance is about $250 \mathrm{WU}$ and is very close to the previous result of $240 \mathrm{WU}$. The numbers of different wall-normal Q2 and Q4 events at this moment are listed in Table 2. It is obvious that Q2 events occur near the wall while Q4 events occur relatively far away from the wall. This phenomenon indicates that it is low speed fluids and high speed fluids that respectively dominate CS near and away from the wall.

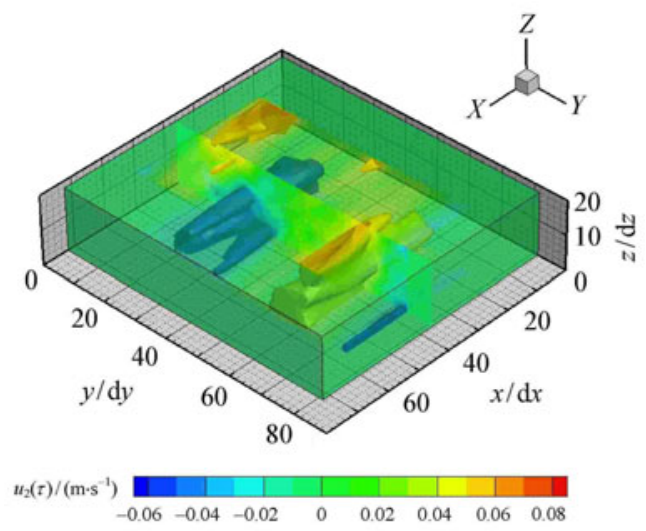

Fig. 6 Isosurface contour of the instantaneous local averaged fluctuating velocity $u_{2}(\tau)$ in log law region

Table 2 The number $N$ of Q2 and Q4 events detected instantaneously at different wall-normal locations

\begin{tabular}{lll|lll}
\hline$z / \mathrm{d} z$ & $\begin{array}{l}N \text { of } \\
\text { Q2 events }\end{array}$ & $\begin{array}{l}N \text { of } \\
\text { Q4 events }\end{array}$ & $z / \mathrm{d} z$ & $\begin{array}{l}N \text { of } \\
\text { Q2 events }\end{array}$ & $\begin{array}{l}N \text { of } \\
\text { Q4 events }\end{array}$ \\
\hline 2 & 108 & 60 & 11 & 39 & 58 \\
3 & 99 & 53 & 12 & 46 & 73 \\
4 & 98 & 39 & 13 & 36 & 82 \\
5 & 98 & 44 & 14 & 27 & 94 \\
6 & 86 & 41 & 15 & 29 & 90 \\
7 & 72 & 49 & 16 & 25 & 71 \\
8 & 57 & 52 & 17 & 28 & 66 \\
9 & 52 & 57 & 18 & 28 & 72 \\
10 & 52 & 52 & 19 & 21 & 75 \\
\hline
\end{tabular}

\subsection{Intrinsic topology extraction}

By setting the locations of Q2 and Q4 events as the center, streamwise Scale 2 and spanwise Scale 3 as length scales of the characteristic space, we have calculated the spatial phase averaging of fluctuating velocity components in the center alignment, and extracted the intrinsic space topology as follows

$\langle\boldsymbol{f}\rangle=\frac{1}{N} \sum_{i \in I} \boldsymbol{f}_{i}$.

In this formula, $f$ indicates the spatial sequence of $u^{\prime} / v^{\prime} / w^{\prime}$, $I$ is the mathematical set of ejection or sweep event locations, and $N$ is the total number of events involved in space phase averaging. We have adopted the suggestion of Schröder et al. [17] that the information of at least 9 layers nearby the events is necessary in all the three directions, and then have made calculations by grouping the measuring points in every 9 wall-normal layers into one box. Therefore, 3D spa- 
tial phase averages with the box size of $32 \mathrm{~d} x \times 16 \mathrm{~d} y \times 9 \mathrm{~d} z$ and center locations of $Z_{+}=115 / 189 / 264$ are conducted, respectively. The results are shown in Fig. 7 and isosurface charts are respectively $u_{\mathrm{e}}=-0.025 \mathrm{~m} / \mathrm{s}$ corresponding to ejection and $u_{\mathrm{s}}=0.035 \mathrm{~m} / \mathrm{s}$ corresponding to sweep. Although based on the same detection function, the space topologies of CS in Figs. 7a-7c are not identical due to the different wall-normal locations of Q2 events and corresponding streaks, whose situaton is similar to the cases of Figs. 7d7f. It is consistent with the results observed in flow visualization experiments by numerous researchers. In velocity vector diagram of cross section $x / \mathrm{d} x=17$, the low speed fluids stretching along streamwise direction are lifted up beside the locations of Q2 events, and the high speed ones are underwashing beside Q4 event locations. The intrinsic space topologies of CS relative to burst events are rendered as the bar-like streak structures with some differences no matter what is the wall-normal location.

Besides the streak structures which own powerful in- termittent fluctuating velocities as their main features, hairpin eddies are the other important kind of CS in log law region and are displayed by strong vortices. The interaction between the two typical structures is mainly in a mutual inductance mechanism. In fact, the place where burst events occur must be a place with large velocity gradient and strong vorticity. Meanwhile, it must be under the hairpin eddy core rotating clockwise and between the two intro-rolling legs of hairpin eddy that streak structures appear. Therefore, the detections of streak structures and hairpin eddies can be based on the same burst events with a possible but slight phase difference. According to the observation of Adrian et al. [9], generalized hairpin eddies may be in various specific topology forms, for example, cane, hairpin, horseshoe, $\Omega$-shape and deformed versions thereof. Compared with the generalized hairpin eddies, calculation of spatial phase averaging to extract characteristic shape is more meaningful for the streak structure whose individual topology has more similar features.
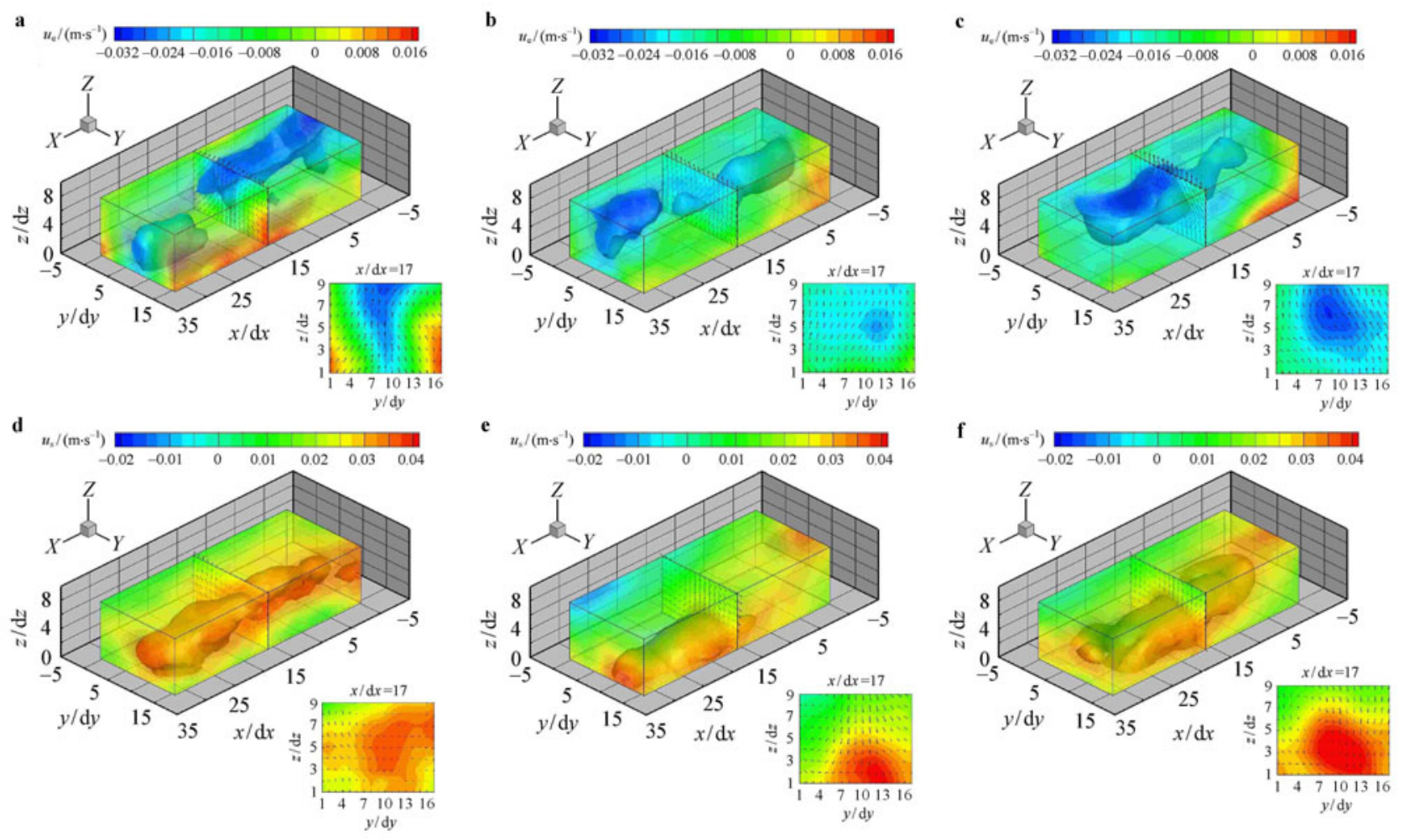

Fig. 7 The spatial topology of streak structures and the velocity fields neighboring the burst events. $\mathbf{a}, \mathbf{b}, \mathbf{c}$ based on Q2 events whose center is at $Z+=115 / 189 / 264 ; \mathbf{d}, \mathbf{e}, \mathbf{f}$ based on $\mathrm{Q} 4$ events whose center is at $Z+=115 / 189 / 264$

\subsection{Spatial-temporal cross-correlation scanning}

Although the streak structures are not invariant in spacetime, they demonstrate a tremendous persistence [31] in their spatial-temporal evolution. When developing downstream, the burst events that produce turbulence are attenuated quickly, while CS often maintain their integrity for a time period up to an order of magnitude longer than the observed bursting time. The spatial-temporal cross-correlation scanning at the fluctuating velocity fields has intrinsic space topology of streak structures as temple. In practice, we calculate the correlation coefficients between the instantaneous streak scalar field $F$ in the spatial box containing $33 \times 17 \times 9$ 
$(x \times y \times z)$ points and the streamwise fluctuating velocity $u^{\prime}$ at adjacent moments.

$$
\begin{aligned}
& R_{F, u^{\prime}}(\Delta x, \Delta y ; \Delta t) \\
& \quad=\frac{\left\langle F(x, y, z ; t) u^{\prime}(x+\Delta x, y+\Delta y, z ; t+\Delta t)\right\rangle}{\left[\left\langle F^{2}(x, y, z ; t)\right\rangle\left\langle u^{\prime 2}(x+\Delta x, y+\Delta y, z ; t+\Delta t)\right\rangle\right]^{1 / 2}},
\end{aligned}
$$

When $F=u_{\mathrm{e}}$, the results are based on Q2 events and when $F=u_{\mathrm{s}}$, they are based on $\mathrm{Q} 4$ events. Since the wall-normal locations of $F$ and $u^{\prime}$ are fixed at the same $z$ without $\Delta z$, the cross-correlation coefficient function has only three variables $(\Delta x, \Delta y, \Delta t)$.

Figure 8 shows the cross-correlation results at $Z+=$ 189, where Fig. 8a is based on Q2 event at $\Delta t / \mathrm{d} t=0$, Fig. $8 \mathrm{~b}$ is based on Q4 event at $\Delta t / \mathrm{d} t=0$, and Fig. $8 \mathrm{c}$ represents the temporal evolution based on Q2 event. In the phase plane $(\Delta x, \Delta y)$, contour lines of cross-correlation contribute in a form of bands, and the maximum and minimum values appear alternately along spanwise direction. The strong correlation areas with relative regular geometrical configuration (ellipse) represent the instantaneous streak structures that are most similar with the intrinsic topology. No matter it is Q2 events or Q4 events, the essence of cross-correlation scanning is statistic analysis on the 2nd order associated physical quantity of velocity, so the positive maximum has a clear physical meaning of the location where cores of streak structures are. To estimate roughly, the positive maximum is moving $40 \mathrm{~d} x$ downstream within $48 \mathrm{~d} t$. The persistence of the streaks meets the requirement of Taylor frozen hypothesis.
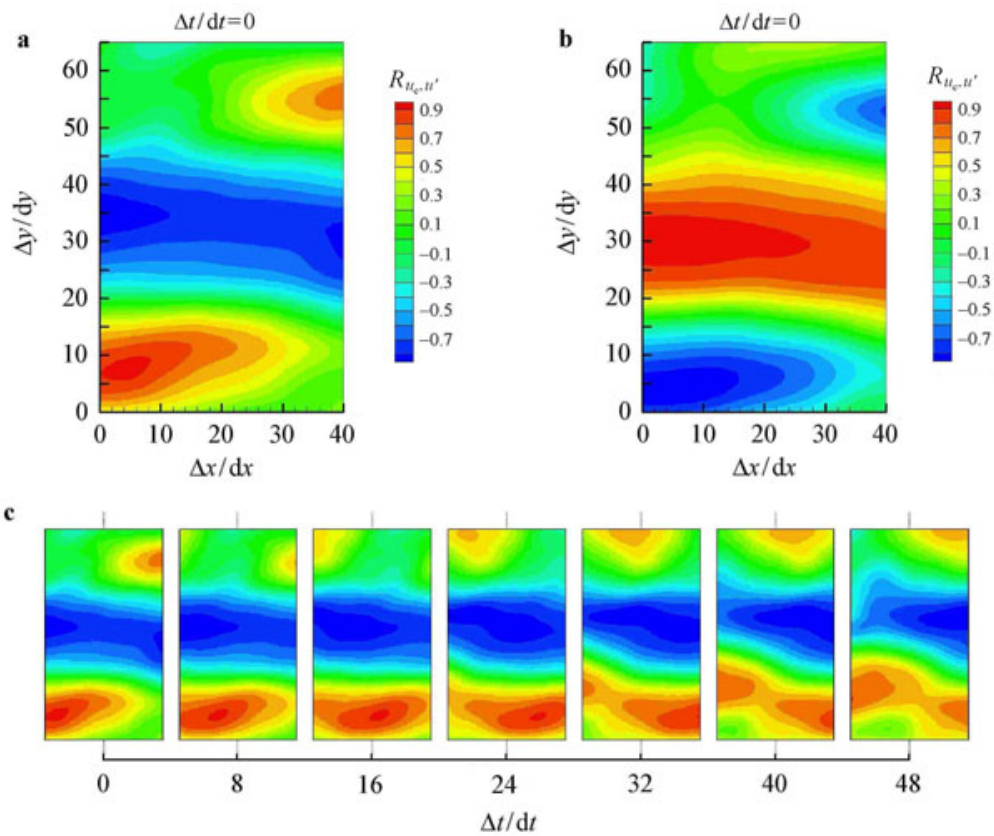

Fig. 8 The spatial-temporal cross-correlation between the intrinsic streak structures $F$ and the stream-wise fluctuating velocity $u^{\prime}$ at $Z+=$ 189. a Based on ejection events $F=u_{\mathrm{e}} ; \mathbf{b}$ Based on sweep events $F=u_{\mathrm{s}}$; $\mathbf{c}$ The temporal evolution based on eject events $F=u_{\mathrm{e}}$

The streak structure moving downstream can be treated as a spatial-temporal function $f(x, t)$. Based on the Taylor frozen hypothesis

$f(x, t)=f\left(x-U_{\mathrm{c}} \times t, 0\right)$,

$U_{\mathrm{c}}$ in this formula is the propagation velocity of the structures. Strong correlation areas also exist in the displacementtime plane $(\Delta x, \Delta t)$ as they do in the displacement phase plane $(\Delta x, \Delta y)$. The line connecting the maximum values is the path line of streak cores, so the slope of this line is just the propagation velocity of the structures. There are three mechanisms when one chooses the magnitude of $\Delta t$ to calculate the propagation velocity [20]. When $\Delta t$ is small enough in the 1st mechanism, according to the definition of limit, the propagation speed of streak structures can be achieved. However, the tremendous changing of $U_{\mathrm{c}}$ with increasing $\Delta t$ demonstrates its dependence on time scale of the structure. From the experimental viewpoint, the 1st mechanism requires a relatively higher time resolution. If the $\Delta t$ is too big, correlation coefficients will be rather small and there must be a huge difference between definitions of the line connecting the maximum values and the path line of streak structures in the $2 \mathrm{nd}$ mechanism. While in the $3 \mathrm{rd}$ mechanism, $\Delta t$ increases appropriately so that the slope of the path line is almost constant corresponding to the locomotory movements of CS.

From Fig. 9 we can see that the streak structure in log law region is moving at a steady speed in streamwise direction and the core of this streak is vibrating slightly in spanwise direction. The cross-correlation coefficients are big 
enough in the range of 0.9-0.93 and conform to Mechanism 3. The comparison results between propagation velocities and mean stream-wise velocities are shown in Fig. 10. The propagation velocities of CS in log law region $Z+=115-$ 264 are as big as $75.6 \%-93 \%$ of the local mean velocities or $53 \%-75 \%$ of the incoming stream velocity. Because of the influence of coming stream, the propagation velocity of streak structure is increased up to a maintenance level of $75 \%$ inflow velocity with increasing distance from the wall.

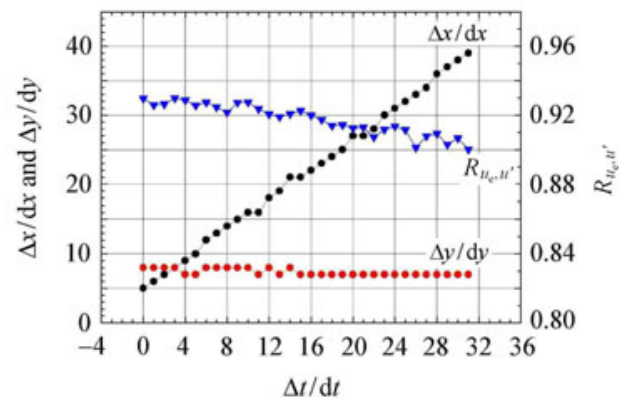

Fig. 9 The magnitude and the locations of the maximum crosscorrelation coefficient at $Z+=189$

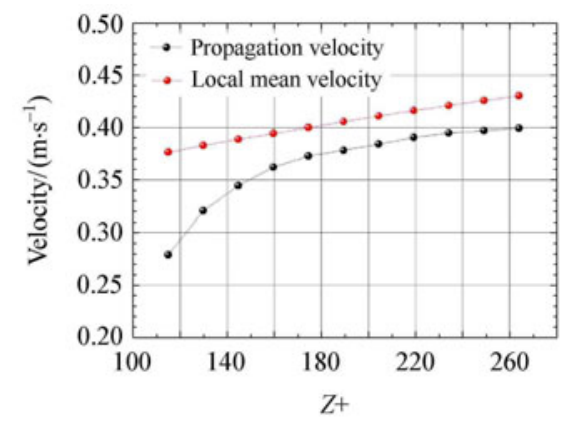

Fig. 10 The propagation velocities of streak structures and the local mean streamwise velocities at different wall-normal locations

\section{Discussion and conclusion}

Newly developed Tomo TRPIV technique facilitates the study on spatial-temporal evolution of the coherent structures. For the 3D-3C velocity signals, to extract intrinsic topology is a pre-request for the study on spatial-temporal evolution of CS, while location identification and size analysis of CS are pre-requests for extracting intrinsic topology.

According to the comparative results of multiresolution wavelet energy at different scale, in the log law region of TBL, we have shown that the stream-wise characteristic length is about $480 \mathrm{WU}$ and the span-wise one of $240 \mathrm{WU}$ is larger than the $100 \mathrm{WU}$ of mean interval between two low speed streaks near wall.

Burst events of ejection and sweep play a significant role in the production of coherent structures. Based on them, results of spatial phase averaging indicate that the CS ex- tracted from physical quantity of fluctuating velocity appear as the bar-like streaks stretching along streamwise direction.

CS are much more persistent than burst events in the space-time evolution which coincides with the Taylor frozen hypothesis. Cross-correlation analysis between fluctuating velocity and CS reflects the distribution and transport property of streak structures. The line connecting the positive maximum values of correlation coefficients is the path line of a streak core in the displacement-time plane $(\Delta x, \Delta t)$. The transport velocity of CS increases with $Z+$ in a range of $75.6 \%-93 \%$ of the local mean velocities in log law region $Z_{+}=115-249$ of TBL for $R_{\theta}=2460$, and finally up to a maintenance level of $75 \%$ of the inflow velocity.

Acknowledgments We are grateful to all the members of the experimental team in Delft University of Technology, LaVision, $\mathrm{GmbH}$ and the German Aerospace Center (DLR) for the Tomo TRPIV dataset of turbulent boundary layer measurement.

\section{References}

1 Kline, S.J., Reynolds, W.C., Schraub F.A., et al.: The structure of turbulent boundary layers. J. Fluid Mech. 30, 741-773 (1967)

2 Robinson, S.K.: Coherent motions in the turbulent boundary layer. Annu. Rev. Fluid Mech. 23, 601-639 (1991)

3 Cantwell, B.J.: Organized motion in turbulent flow. Annu. Rev. Fluid Mech. 13, 457-515 (1981)

4 Hussain, A.: Coherent structures and turbulence. J. Fluid Mech. 173, 303-356 (1986)

5 Perry, A.E., Chong, M.S.: On the mechanism of wall turbulence. J. Fluid Mech. 119, 173-217 (1982)

6 Panton, R.L.: Overview of the self-sustaining mechanisms of wall turbulence. Prog. Aerosp. Sci. 37, 341-384 (2001)

7 Balakumar, B.J., Adrian, R.J.: Large- and very-large-scale motions in channel and boundary-layer flows. Philos. Trans. R. Soc. Lond Ser. A Math. Phys. Eng. Sci. 365, 665-681 (2007)

8 Falco, R.E.: Coherent motions in the outer region of turbulent boundary layers. Physics of Fluids 20, 124-132 (1997)

9 Adrian, R.J., Meinhart, C.D., Tomkins, C.D.: Vortex organization in the outer region of the turbulent boundary layer. J. Fluid Mech. 422, 1-54 (2000)

$10 \mathrm{Lu}$, S.S., Willmarth, W.W.: Measurements of the structure of the Reynolds stress in a turbulent boundary layer. J. Fluid Mech. 60, 481-511 (1973)

11 Sun, K.H., Shu, W.: On the burst detection techniques in wallturbulence. Chin. J. Theor. Appl. Mech. 26, 488-493 (1994) (in Chinese)

12 Ganapathisubramani, B., Longmire, E.K., Marusic, I.: Characteristics of vortex packets in turbulent boundary layers. J. Fluid Mech. 478, 35-46 (2003)

13 Kähler, C.J.: The significance of coherent flow structures for the turbulent mixing in wall-bounded flows. Dissertation, DLR Forschungsbericht, 2004-24, ISSN 1434-8454 (2004)

14 Adrian, R.J.: Twenty years of particle image velocimetry. Exp. Fluids 39, 159-169 (2005)

15 Tang, Z.Q., Jiang, N.: TR PIV experimental investigation on bypass transition induced by a cylinder wake. Chin. Phys. Lett. 28, 054702 (2011) 
16 Schröder, A., Geisler, R., Elsinga, G.E., et al.: Investigation of a turbulent spot and a tripped turbulent boundary layer flow using time-resolved tomographic PIV. Exp. Fluids 44, 305-316 (2008)

17 Schröder, A., Geisler, R., Elsinga, G.E., et al.: Eulerian and Lagrangian views of a turbulent boundary layer flow using timeresolved tomographic PIV. Exp. Fluids 50, 1071-1091 (2011)

18 Yang, S.Q., Jiang, N.: Tomographic TR-PIV measurement of coherent structure spatial topology utilizing an improved quadrant splitting method. Science China 55, 1863-1872 (2012)

19 del Alamo, J.C., Jiménez, P.Z.: Estimation of turbulent convection velocities and corrections to Taylor's approximation. J. Fluid Mech. 640, 5-26 (2009)

20 Kim, J., Hussain, F.: Propagation velocity of perturbations in turbulent channel flow. Physics of Fluids A5, 695-701 (1993)

21 Chang, S., Blackwelder, R.F.: Modification of large eddies in turbulent boundary layers. J. Fluid Mech. 213, 419-442 (1990)

22 Jacobson, S.A., Reynolds, W.C.: Active control of streamwise vortices and streaks in boundary layers. J. Fluid Mech. 360, 179-211 (1998)

23 Novara, M., Batenburg, K.J, Scarano, F.: Motion trackingenhanced MART for tomographic PIV. Meas. Sci. Technol. 21, 1-18 (2010)
24 Wieneke, B.: Volume self-calibration for stereo PIV and tomographic PIV. In: Proceedings of PIV'07. Rome, 11-14 (2007)

25 Liu, J.H., Jiang, N., Wang, Z.D., et al.: Multi-scale coherent structures in turbulent boundary layer detected by locally averaged velocity structure functions. Applied Mathematics and Mechanics 26, 456-464 (2005)

26 Jiang, N., Zhang, J.: Detecting multi-scale coherent eddy structures and intermittency in turbulent boundary layer by wavelet analysis. Chin. Phys. Lett. 22, 1968-1971 (2005)

27 Mallat, S.G.: A theory for multiresolution signal decomposition: the wavelet representation. IEEE Transactions on Pattern Analysis and Machine Intelligence 11, 674-693 (1989)

28 Farge, M., Schneider, K.: Coherent Vortex Simulation (CVS), a semideterministic turbulence model using wavelets. Flow Turbul. Combust. 66, 393-426 (2001)

29 Jiang, N., Wang, Z.D., Shu, W.: The maximum energy criterion for identifying burst events in wall turbulence using wavelet analysis. Acta Mechanica Sinica 29, 406-411 (1997) (in Chinese)

30 Liu, W., Jiang, N.: Three kinds of velocity structure function in turbulent flows. Chin. Phys. Lett. 21, 1989-1992 (2004)

31 Smith, C.R., Metzler, S.P.: The characteristics of low-speed streaks in the near-wall region of a turbulent boundary layer. J. Fluid Mech. 129, 27-54 (1983) 